J. Chosun Natural Sci.

Vol. 7, No. 1 (2014) pp. $45-49$

http://dx.doi.org/10.13160/ricns.2014.7.1.45

\title{
A CoMFA Study of Phenoxypyridine-Based JNK3 Inhibitors Using Various Partial Charge Schemes
}

\author{
Pavithra K. Balasubramanian ${ }^{1 \dagger}$, Anand Balupuri ${ }^{1 \dagger}$ and Seung Joo Cho ${ }^{1,2 \dagger}$ \\ Abstract
}

The (c-Jun N-terminal kinase 3) JNK3 is a potential therapeutic target for various neurological disorders. Here, a three dimensional quantitative structure-activity relationship (3D-QSAR) study on phenoxypyridine as JNK3 inhibitors was performed to rationalize the structural requirements responsible for the inhibitory activity of these compounds. The comparative molecular field analysis (CoMFA) using different partial atomic charges, was employed to understand the structural factors affecting JNK3 inhibitory potency. The Gasteiger-Marsili yielded a CoMFA model with cross-validated correlation coefficient $\left(\mathrm{q}^{2}\right)$ of 0.54 and non-cross-validated correlation coefficient $\left(\mathrm{r}^{2}\right)$ of 0.93 with five components. Furthermore, contour maps suggested that bulky substitution with oxygen atom in $\mathrm{R}^{3}$ position could enhance the activity considerably. The work suggests that further chemical modifications of the compounds could lead to enhanced activity and could assist in the design of novel JNK3 inhibitors.

Key words: 3D-QSAR, CoMFA, JNK3, Phenoxypyridine Derivatives

\section{Introduction}

The c-Jun N-terminal kinases (JNKs) are serine/threonine protein kinases and belong to the mitogen-activated protein kinase family (MAPK). The JNKs can be activated in response to various stimuli such as cytokines, mitogens, osmotic stress and ultraviolet irradiation. Activated JNKs lead to the phosphorylation of several transcription factors related to apoptosis ${ }^{[1-3]}$. Of the three isoforms (JNK1, 2, and 3), JNK3 is expressed primarily in the brain and plays important role in central nervous system (CNS) disorders. Dysfunctions of JNK3 have been implicated in the etiology of a variety of neurological disorders such as Parkinson's disease, Alzheimer's disease, multiple sclerosis, epilepsy and stroke. JNK3 is a suitable therapeutic target for neurodegenerative diseases such as Alzheimer's and Parkinson's diseases in addition to Huntington's disease, and cerebral ischemia $^{[4-6]}$.

In the recent years, several compounds have been

${ }^{1}$ Departments of 1Bio-New Drug Development and ${ }^{2}$ Cellular.Molecular Medicine, College of Medicine, Chosun University, Gwangju 501-759, Republic of Korea

†Corresponding author: pavithrabioinfo@gmail.com,

anandbalupuri.niper@gmail.com, chosj@chosun.ac.kr

(Received: March 5, 2014, Revised : March 13, 2014,

Accepted : March 25, 2014) reported as JNK3 inhibitors. Although many of them possess potent in vitro activities, they often lack selectivity or have weak in vivo potency. Thus, developing successful drugs targeting JNK3 is still a challenge. Previously our group has reported several papers covering different in silico applications such as docking, 3DQSAR and importance of partial charges ${ }^{[7-12]}$. Here, 3DQSAR analysis was performed on the series of 29 phenoxypyridine inhibitors ${ }^{[13]}$. Influence of different charge schemes on the CoMFA models was investigated. The relationship between structure and activity of the inhibitors suggested structural features contributing to the inhibitory potency. This study would guide the design of new potent JNK3 inhibitors.

\section{Experimental Section}

\subsection{Data Set}

A data set of 29 phenoxypyridine derivatives was collected from the previous work ${ }^{[13]}$. The reported $\mathrm{IC}_{50}$ values for all compounds were converted into $\mathrm{pIC}_{50}\left(-\log \mathrm{IC}_{50}\right)$, in order to use the data as the dependent variable in the CoMFA model. The chemical structures and biological activities of the inhibitors are listed in Table 1. All the 3D structures of the compounds were constructed using Sketch program in Sybylx2.0 $0^{[14]}$ and 
Table 1. Structures and biological activities of phenoxypyridine derivatives

\begin{tabular}{|c|c|c|c|c|}
\hline \multicolumn{2}{|c|}{ Compound $9 \mathrm{a}-9 \mathrm{c}$} & \multirow[b]{2}{*}{$\mathrm{R}^{3}$} & \multicolumn{2}{|c|}{ Compound $10 \mathrm{a}-10 \mathrm{q} \& 11 \mathrm{a}-11 \mathrm{i}$} \\
\hline Compound & $\mathrm{R}^{1}$ & & $\mathrm{R}^{2}$ & $\mathrm{pIC}_{50}$ \\
\hline $9 \mathrm{a}$ & $\mathrm{F}$ & & - & 7.319 \\
\hline $9 \mathrm{~b}$ & $\mathrm{Cl}$ & & - & 7.824 \\
\hline $9 \mathrm{c}$ & $\mathrm{CF}_{3}$ & & - & 7.432 \\
\hline $10 \mathrm{a}$ & - & 3-MeOPh & - & 7.155 \\
\hline $10 \mathrm{~b}$ & - & 2-MeOPh & - & 5.456 \\
\hline $10 \mathrm{c}$ & - & $4-\mathrm{CF}_{3} \mathrm{OPh}$ & - & 7.456 \\
\hline $10 \mathrm{~d}$ & - & 4-EtOPh & - & 6.959 \\
\hline $10 \mathrm{e}$ & - & 4- $n$-PrOPh & - & 6.854 \\
\hline $10 \mathrm{f}$ & - & $4-t-\mathrm{BuOPh}$ & - & 7.569 \\
\hline $10 \mathrm{~g}$ & - & 4-FPh & - & 6.699 \\
\hline $10 \mathrm{~h}$ & - & 4-BrPh & - & 6.420 \\
\hline $10 \mathrm{i}$ & - & 3-ClPh & - & 6.699 \\
\hline $10 \mathrm{j}$ & - & 3,4-ClPh & - & 7.000 \\
\hline $10 \mathrm{k}$ & - & 3,4-Cl,4-MeOPh & - & 7.000 \\
\hline 101 & - & 1-Naphthyl & - & 6.004 \\
\hline $10 \mathrm{~m}$ & - & 2-Naphthyl & - & 6.495 \\
\hline $10 n$ & - & 3,4-MethylenedioxyPh & - & 7.319 \\
\hline $10 \mathrm{o}$ & - & 4-Triazole-Ph & - & 7.097 \\
\hline $10 \mathrm{p}$ & - & 4-Piperazine-Ph & - & 7.469 \\
\hline $10 q$ & - & 3-Pyridyl-4-Ph & - & 7.143 \\
\hline $11 \mathrm{a}$ & - & - & $2-\mathrm{Cl}$ & 5.658 \\
\hline $11 b$ & - & - & 2-OMe & 5.658 \\
\hline $11 \mathrm{c}$ & - & - & $3-\mathrm{F}$ & 7.347 \\
\hline $11 d$ & - & - & $3-\mathrm{Cl}$ & 7.523 \\
\hline $11 \mathrm{e}$ & - & - & $4-\mathrm{F}$ & 7.310 \\
\hline $11 \mathrm{f}$ & - & - & $4-\mathrm{Cl}$ & 7.108 \\
\hline $11 \mathrm{~g}$ & - & - & 4-OMe & 7.770 \\
\hline $11 \mathrm{~h}$ & - & - & $5-\mathrm{F}$ & 7.222 \\
\hline $11 \mathrm{i}$ & - & - & $5-\mathrm{Cl}$ & 6.796 \\
\hline
\end{tabular}

their energies were minimized using Tripos force field. Most active inhibitor (9b) was selected as the template for sketching and alignment of the all other compounds. The lowest energy conformation of template molecule

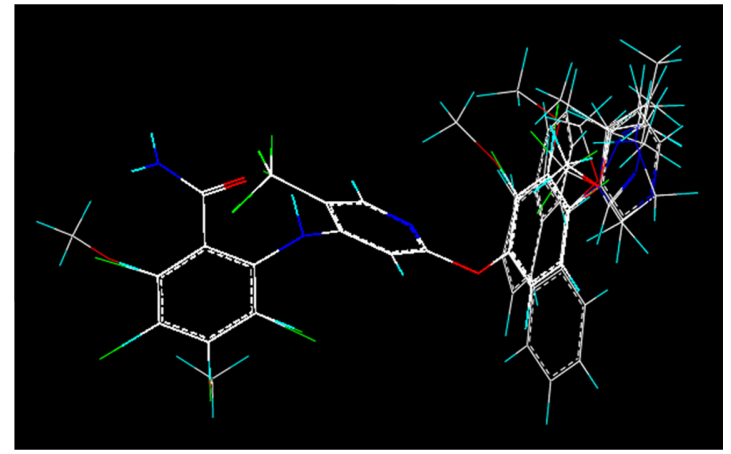

Fig. 1. Alignment of data set molecules based on common substructure using template molecule $(9 \mathrm{c})$.

obtained from systematic conformational search was used for sketching the rest of the molecules by changing the substituents. The common moiety was constraint for each molecule and only the varying parts were energy minimized using Tripos force field. The minimized structures were aligned over the template using common substructure based alignment method. The aligned compounds are displayed in Fig. 1.

\subsection{CoMFA}

The CoMFA method was developed by Cramer et $a l^{[15]}$. CoMFA calculations were carried out by applying the default settings. Aligned molecules were placed in the $3 \mathrm{D}$ cubic lattice with the grid spacing of 2.0 A. Electrostatic and steric fields in CoMFA were calculated from Coulomb and Lennard-Jones potentials, respectively. The fields were generated using $\mathrm{sp}^{3}$ carbon probe atom carrying +1 charge and van der Waals radius of $1.50 \AA$. Energy cut-off value of $30 \mathrm{kcal} \mathrm{mol}^{-1}$ was adopted for both fields.

The relationship between the structural parameters and the biological activities was derived by the partial least squares (PLS) regression algorithm ${ }^{[16]}$. CoMFA descriptors were used as independent variables and $\mathrm{pIC}_{50}$ values were used as dependent variables in the PLS analysis. Cross-validation analysis was performed using the leave one out (LOO) method to determine the optimum number of components (ONC) with minimum standard error of prediction (SDEP). In this procedure, one compound was removed from the data set and its activity was predicted using the model derived from the rest of the molecules. The cross-validated correlation coefficient $\left(\mathrm{q}^{2}\right)$ that resulted in the ONC and lowest 
SDEP were considered for further analysis. The noncross-validated analysis was performed to determine conventional Pearson correlation coefficient $\left(\mathrm{r}^{2}\right)$, standard error of estimate (SEE) and Fischer's ratio (F) using the $\mathrm{ONC}$ previously obtained from the cross-validation method.

\section{Results and Discussion}

\subsection{CoMFA Analysis}

CoMFA models were developed using a series of derivatives possessing inhibitory activity against JNK3. Since only 29 compounds were available for model generation, data set was not divided into training and test set. We employed ligand based alignment scheme for generating COMFA models. Alignment was carried out using the most active compound (9b) as a template. We tested various charge schemes during CoMFA modeling. Developed models produced statistically reasonable results which are summarized in Table 2. These results were obtained at a column filtering of $2 \mathrm{kcal}$ $\mathrm{mol}^{-1}$ for both steric and electrostatic fields. GasteigerMarsili charge scheme produced more reliable CoMFA model in terms of several statistical parameters and was selected as final model. The $\mathrm{q}^{2}$ value was selected to determine ONC. PLS analysis showed a high $\mathrm{q}^{2}$ value of 0.54 with five components. The non-cross-validated analysis produced $r^{2}$, SEE and $F$ values of $0.93,0.18$ and 62.96 , respectively. The correlation between actual and predicted values (Table 3) from the selected CoMFA model is shown in Fig. 2.

\subsection{CoMFA Contour Maps}

The CoMFA results were graphically interpreted by the field contribution maps using the $\mathrm{STDEV} * \mathrm{COEFF}$
Table 3. Actual and predicted activities of compounds by the selected CoMFA model

\begin{tabular}{cccc}
\hline Compound & $\begin{array}{c}\text { Actual } \\
\text { pIC }_{50}\end{array}$ & $\begin{array}{c}\text { Predicted } \\
\text { pIC }_{50}\end{array}$ & Residual \\
\hline $10 \mathrm{a}$ & 7.155 & 7.274 & -0.119 \\
$10 \mathrm{~b}$ & 5.456 & 5.266 & 0.19 \\
$10 \mathrm{c}$ & 7.456 & 7.246 & 0.21 \\
$10 \mathrm{~d}$ & 6.959 & 6.877 & 0.082 \\
$10 \mathrm{e}$ & 6.854 & 6.734 & 0.12 \\
$10 \mathrm{f}$ & 7.569 & 7.533 & 0.036 \\
$10 \mathrm{~g}$ & 6.699 & 6.767 & -0.068 \\
$10 \mathrm{~h}$ & 6.420 & 6.657 & -0.237 \\
$10 \mathrm{i}$ & 6.699 & 6.668 & 0.031 \\
$10 \mathrm{j}$ & 7.000 & 7.032 & -0.032 \\
$10 \mathrm{k}$ & 7.000 & 6.977 & 0.023 \\
$10 \mathrm{l}$ & 6.004 & 5.927 & 0.077 \\
$10 \mathrm{~m}$ & 6.495 & 6.597 & -0.102 \\
$10 \mathrm{n}$ & 7.319 & 7.326 & -0.007 \\
$10 \mathrm{o}$ & 7.097 & 7.193 & -0.096 \\
$10 \mathrm{p}$ & 7.469 & 7.450 & 0.019 \\
$10 \mathrm{q}$ & 7.143 & 7.170 & -0.027 \\
$11 \mathrm{a}$ & 5.658 & 5.932 & -0.274 \\
$11 \mathrm{~b}$ & 5.658 & 5.595 & 0.063 \\
$11 \mathrm{c}$ & 7.347 & 7.410 & -0.063 \\
$11 \mathrm{~d}$ & 7.523 & 7.552 & -0.029 \\
$11 \mathrm{e}$ & 7.310 & 7.415 & -0.105 \\
$11 \mathrm{f}$ & 7.108 & 7.145 & -0.037 \\
$11 \mathrm{~g}$ & 7.770 & 7.473 & 0.297 \\
$11 \mathrm{~h}$ & 7.222 & 7.232 & -0.01 \\
$11 \mathrm{i}$ & 6.796 & 7.114 & -0.318 \\
$9 \mathrm{a}$ & 7.319 & 7.543 & -0.224 \\
$9 \mathrm{~b}$ & 7.824 & 7.460 & 0.364 \\
$9 \mathrm{c}$ & 7.432 & 7.190 & 0.242 \\
\hline & & & \\
\hline
\end{tabular}

Table 2. Statistical summary of the developed CoMFA models with different charge schemes

\begin{tabular}{cccccccc}
\hline Parameter & $\begin{array}{c}\text { Gasteiger- } \\
\text { Huckel }\end{array}$ & $\begin{array}{c}\text { Gasteiger- } \\
\text { Marsili }\end{array}$ & $\begin{array}{c}\text { Formal } \\
\text { charges }\end{array}$ & MMFF94 & Del-re & Huckel & Pullman \\
\hline $\mathrm{q}^{2}$ & 0.45 & $\mathbf{0 . 5 4}$ & 0.43 & 0.46 & 0.57 & 0.43 & 0.43 \\
$\mathrm{~N}$ & 5 & $\mathbf{5}$ & 5 & 4 & 4 & 6 & 5 \\
$\mathrm{SEP}$ & 0.51 & $\mathbf{0 . 4 7}$ & 0.52 & 0.49 & 0.44 & 0.53 & 0.52 \\
$\mathrm{r}^{2}$ & 0.96 & $\mathbf{0 . 9 3}$ & 0.96 & 0.95 & 0.91 & 0.97 & 0.97 \\
$\mathrm{SEE}$ & 0.14 & $\mathbf{0 . 1 8}$ & 0.14 & 0.15 & 0.21 & 0.13 & 0.12 \\
$\mathrm{~F}$ & 105.82 & $\mathbf{6 2 . 9 6}$ & 110.87 & 113.11 & 57.07 & 112.06 & 128.02 \\
\hline
\end{tabular}

$\mathrm{q}^{2}=$ cross-validated correlation coefficient; $\mathrm{N}=$ number of components; $\mathrm{SEP}=$ standard error of prediction; $\mathrm{r}^{2}=$ correlation coefficient; SEE $=$ standard error of estimate; $F=$ F-ratio. 


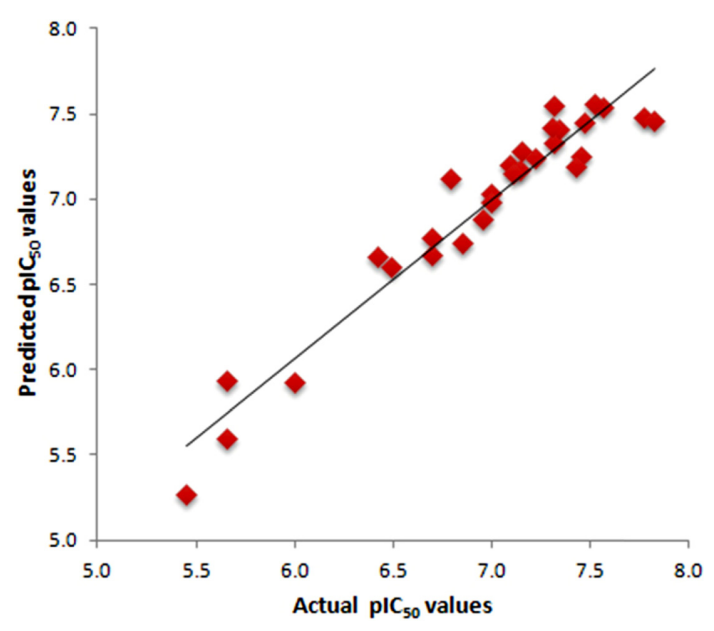

Fig. 2. Scatter plot diagram of predicted versus actual activities of compounds by CoMFA analysis.

field type. The contour maps of different field contribution of the model are displayed with template molecule (9b) and depict default $80 \%$ and $20 \%$ level contributions for favorable and unfavorable regions, respectively. The maps display spatial requirement of steric and electrostatics field for enhancing the inhibitory activity. The Steric contour map for the selected CoMFA model is shown in Fig. 3. The green color signifies regions that favor sterically bulky groups and yellow color signifies the unfavorable regions for bulky substitution. A big green contour was seen in the $\mathrm{R}^{3}$ position of the phenyl ring suggested that bulky substitution in this region is favorable to enhance the activity. This might the possible reason for better inhibitory activity of compounds 10f, 10n, 10o, 10p and 10q which possess long substituents in that position. The two small yellow contours observed near the $\mathrm{R}^{2}$ region of the phenyl ring shows that bulky substituents in this region could reduce the activity. This could be the possible reason for the lower activity of compounds 11a which possess a long substitution in that position.

The electrostatic contour map of the selected CoMFA model is shown in Fig. 4. The blue color signifies positive charge is favored and red color signifies that negative charge is favored to increase the potency. The small blue contour seen near the $\mathrm{R}^{2}$ substitution suggests that positive substitution in that position could increase the activity of ligands. The small red contour seen near the oxygen atom of the $\mathrm{R}^{3}$ position suggests that the substitution that have negative group in that par-

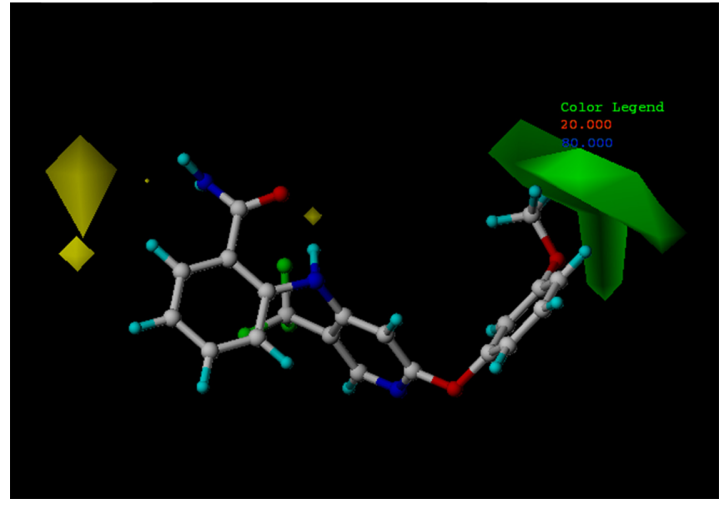

Fig. 3. Contour map for steric field: favored (green) and disfavored (yellow).

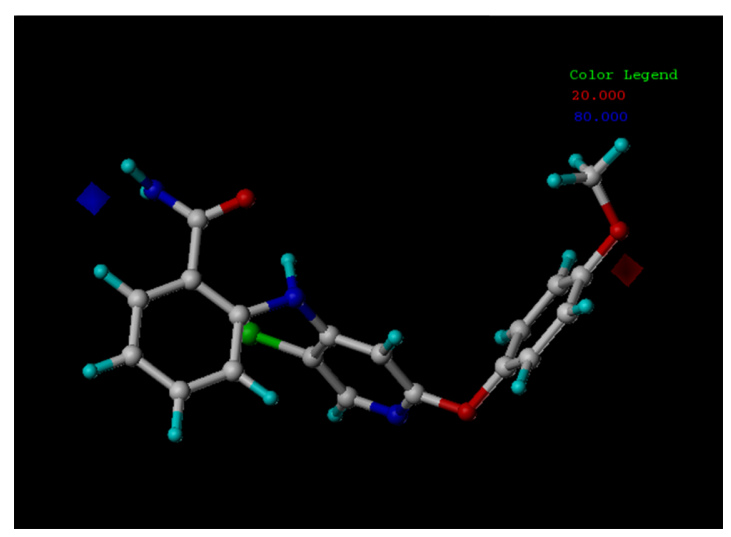

Fig. 4. Contour map for electrostatic field: electropositive (blue) and electronegative (red).

ticular position could increase the activity. This could be the reason why compounds $10 \mathrm{c}, 10 \mathrm{f}$ and $10 \mathrm{n}$ containing oxygen atom in that position have exhibited increased activity. On the other hand, compounds $10 \mathrm{~g}, 10 \mathrm{~h}, \mathbf{1 0} \mathbf{i}$ and $\mathbf{1 0 l}$ not containing oxygen atom in that position has lower activity levels. Thus, the contour map highlights the favorable regions to increase the potency of the ligands.

\section{Conclusions}

In this work, 3D-QSAR models were developed for the series of JNK3 inhibitors. We applied various charge schemes to generate a reasonable CoMFA model. On the basis of the ligand-based alignment and GasteigerMarsili charges, a reliable CoMFA model was obtained. The model showed satisfactory predictive abilities in 
terms of $\mathrm{q}^{2}$ and $\mathrm{r}^{2}$. Contour map analysis suggested that bulky substitution with oxygen atom in $\mathrm{R}^{3}$ position could enhance the activity considerably. Similarly, small positive substitutions in $\mathrm{R}^{2}$ position could increase the activity. These results may provide some useful and rational suggestions for further design of novel JNK3 inhibitors.

\section{Acknowledgements}

This research was supported by Basic Science Research Program through the National Research Foundation of Korea (NRF) funded by the Ministry of Education, Science and Technology (NRF-2012R1A1A4A01001465).

\section{References}

[1] V. Adler, A. Polotskaya, F. Wagner, and A. S. Kraft, "Affinity purified c-Jun amino-terminal protein kinase requires serinethreonine phosphorylation for activity", J. Biol. Chem., Vol. 267, pp. 1700117005, 1992.

[2] R. J. Davis, "Signal transduction by the JNK group of MAP kinases", Cell, Vol. 103, pp. 239-252, 2000.

[3] G. Pearson, F. Robinson, T. Beers, B. Gibson, E. $\mathrm{Xu}, \mathrm{M}$. Karandikar, K. Berman, and M. H. Cobb, "Mitogen-activated protein (MAP) kinase pathways: regulation and physiological functions", Endocr. Rev., Vol 22, pp. 153-183, 2001.

[4] E. K. Kim and E. J. Choi, "Pathological roles of MAPK signaling pathways in human diseases", BBA-Mol. Basis Dis., Vol. 1802, pp. 396-405, 2010.

[5] B. Salh, "c-Jun N-terminal kinases as potential therapeutic targets", Expert Opin. Ther. Targets, Vol. 11, pp. 1339-1353, 2007.

[6] T. Borsello and G. Forloni, "JNK signalling: a possible target to prevent neurodegeneration", Curr. Pharm. Des., Vol. 13, pp. 1875-1886, 2007.

[7] C. G. Gadhe, G. Kothandan, and S. J. Cho, "Large variation in electrostatic contours upon addition of steric parameters and the effect of charge calculation schemes in CoMFA on mutagenicity of MX analogues", Mol. Simulat., Vol. 38, pp. 861-871, 2012.

[8] T. Madhavan, C. G. Gadhe, G. Kothandan, K. Lee, and S. J. Cho, "Various atomic charge calculation schemes of CoMFA on HIF-1 inhibitors of moracin analogs", Int. J. Quantum Chem., Vol. 112, pp. 995$1005,2012$.

[9] A. Balupuri and S. J. Cho, "Exploration of the binding mode of indole derivatives as potent HIV-1 inhibitors using molecular docking simulations", J. Chosun Natatural Sci., Vol. 6, pp. 138-142, 2013.

[10] P. K. Balasubramanian and S. J. Cho, "HQSAR analysis on novel series of 1-(4-phenylpiperazin-1yl-2-(1H-pyrazol-1-yl) ethanone derivatives targeting CCR1", J. Chosun Natural Sci., Vol. 6, pp. 163169, 2013.

[11] S. J. Cho, "Calculation and application of partial charges", J. Chosun Natural Sci., Vol. 3, pp. 226230, 2010.

[12] S. J. Cho, "Meaning and definition of partial charges", J. Chosun Natural Sci., Vol. 3, pp. 231236, 2010.

[13] X. Song, W. Chen, L. Lin, C. H. Ruiz, M. D. Cameron, D. R. Duckett, and T. M. Kamenecka, "Synthesis and SAR of 2-Phenoxypyridines as novel cJun N-terminal kinase inhibitors", Bioorg. Med. Chem. Lett., Vol. 21, pp. 7072-7075, 2011.

[14] Tripos Associates, SYBYL X Molecular Modeling Software, Version 1.2 (2011), Tripos Associates, St. Louis, MO (http://www.tripos.com).

[15] R. D. Cramer, D. E. Patterson, and J. D. Bunce, "Comparative molecular field analysis (CoMFA). 1. Effect of shape on binding of steroids to carrier proteins", J. Am. Chem. Soc., Vol. 110, pp. 5959-5967, 1988.

[16] S. Wold, A. Ruhe, H. Wold, and I. W. Dunn, "The collinearity problem in linear regression. The partial least squares (PLS) approach to generalized inverses", SIAM J. Sci. Comput., Vol. 5, pp 735743, 1984. 